\title{
DERECHOS HUMANOS, DESAPARICIÓN FORZADA Y FEMINICIDIO EN EL ESTADO DE Chinuahua
}

\section{RESUMEN}

$\Gamma$ a violencia ejercida contra las mujeres constituye una ofensa a la dignidad de las personas y una violación a los derechos humanos; se ha convertido en un problema social, económico, jurídico, sanitario y político de alcance mundial y una manifestación retrógrada al reproducir relaciones de poder desiguales entre hombres y mujeres, cuyas máximas expresiones son la desaparición forzada y el feminicidio. Si bien en México y en el estado de Chihuahua se identifican avances significativos en materia de derechos humanos, dicho tipo de violencia se ha incrementado considerablemente en las últimas décadas, contabilizándose en centenas los casos registrados anualmente.

Palabras clave: Derechos humanos de las mujeres, violencia contra las mujeres, estado de Chihuahua.

1 Profesora investigadora Cátedra Conacyt, adscrita a El Colegio de la Frontera Norte. Chihuahua Hoy, año 18, Núm. 18 (enero-diciembre, 2020): 


\begin{abstract}
Violence against women constitutes an offense to the dignity of people and a violation of human rights has become a global social, economic, legal, health and political problem and a retrograde manifestation by reproducing unequal power relations between men and women, whose maximum expressions are enforced disappearance and femicide. While significant progress in human rights is identified in Mexico and the state of Chihuahua, significant human rights progress is identified, this type of violence has increased considerably in recent decades, hundreds of cases recorded annually.
\end{abstract}

Key Words: Human rights of women, Violence against women, Chihuahua State.

\title{
INTRODUCCIÓN
}

La violencia ejercida contra las mujeres -y niñas - es una expresión de la desigualdad de género, que se ha convertido en un problema social, económico, jurídico, sanitario y político de alcance mundial (ONU Mujeres, 2020), cuyas expresiones más radicales y dramáticas son la desaparición forzada y el feminicidio, y que tan solo de estos últimos se contabilizan anualmente más de 66000 a nivel mundial. En 2016 en América Latina se registró un promedio de 12 feminicidios al día, destacando que seis de ellos sucedieron en México (Naciones Unidas, CEPAL, 2016), cifra que se aproxima a la expuesta en el documento Violencia Feminicida en México, que señala que son más de 7 las victimadas cada 24 horas (ONU Mujeres, Secretaría de Gobernación, Instituto Nacional de las Mujeres, 2017).

México atraviesa por una grave crisis de violaciones de derechos humanos. Tan solo en el periodo 2006-2015 el gobierno mexicano recibió 548 recomendaciones en dicha materia por parte de organismos internacionales, debido a los altos índices de violencia que se traducen en casos de tortura, ejecuciones extrajudiciales, 
abuso a migrantes, desapariciones forzadas y feminicidios, principalmente (Carbajal et al., 2019), cifra elevada para un país que oficialmente no se encuentra en un conflicto armado (Román, 2015).

Durante 2019 en México perdieron la vida de forma violenta más de 40000 personas —convirtiéndose en el año más violento-, de las cuales más de 900 se tipificaron como feminicidios, generalizándose el problema en 385 municipios (Secretariado Ejecutivo del Sistema Nacional de Seguridad Pública, 2019). No obstante, es necesario puntualizar que otros registros refieren que para ese mismo año a nivel nacional se contabilizaron alrededor de 4000 defunciones de mujeres por causas violentas -feminicidios-, de las cuales más de 300 se presentaron en el estado de Chihuahua (Inegi, 2019).

En cuanto a la violencia ejercida contra las mujeres en la modalidad de desaparición forzada, no es posible siquiera cuantificar puntualmente los casos, ya que si bien existe una Comisión Nacional de Búsqueda de Personas, desde 2018 dejó de alimentarse el Registro Nacional de Personas Extraviadas o Desaparecidas, derivando en la ausencia de datos oficiales relativos. Sin embargo, informes periodísticos estiman que en México son más de 61000 personas desaparecidas de 2006 a la fecha (Villegas, 2020), evidenciando con ello la grave crisis de violencia que prevalece en el país.

Principalmente en las ciudades fronterizas de México se identifica una importante presencia de grupos del crimen organizado y la violencia se ha incrementado exponencialmente durante los últimos 25 años, provocando un importante y generalizado deterioro de los niveles de seguridad y bienestar de la población (Díaz \& Reséndiz, 2017). Cifras oficiales destacan los altos índices de violencia que privan en el estado de Chihuahua y que simplemente de muertes violentas concentra el 20\% de los 8000 casos ocurridos a nivel nacional (Inegi, 2019), donde las principales víctimas son hombres pobres y jóvenes, que con frecuencia son pandilleros o reclutados como narcomenudistas o sicarios (Caballero, 2011; Cruz, 2014). 
Si bien la violencia la padecen tanto hombres como mujeres, dados los esquemas culturales y sociales es necesario puntualizar en la ejercida contra las mujeres (Masters, 2009), de ahí que se observe cómo destaca en el estado de Chihuahua este tipo de violencia, ocupando la sexta posición a nivel nacional, solamente por debajo de la Ciudad de México, el Estado de México, Jalisco y Aguascalientes (Inegi, 2016). Algunos medios refieren que la cifra de feminicidios ocurridos solamente en Ciudad Juárez, entre 1993 a 2010, superó el medio millar (BBC News Mundo, 2016); sin embargo, en las bases de datos generadas por centros de investigación, como la de El Colegio de la Frontera Norte, se observa que en dicho estado la violencia contra las mujeres - del tipo de desaparición forzada y feminicidio - se recrudece a partir de las dos últimas décadas del siglo pasado, contabilizándose por cientos los casos registrados, de los cuales la mayoría sigue sin esclarecerse, sin identificarse a los culpables y consecuentemente sin castigarlos (Monárrez, 2004; Rodríguez, 2005), incrementándose al grado de ser considerado un exterminio (Monárrez, 2010).

La violencia ejercida contra las mujeres constituye una ofensa contra la dignidad humana y una manifestación retrógrada, al reproducir relaciones de poder desigual entre hombres y mujeres (Alberdi \& Matas, 2002). Los derechos comúnmente son limitados, cuestionados o negados, tanto en contextos locales como globales (Faci \& Victory, 2017), por lo que se consideran una violación a los derechos humanos de estas, que se traducen total o parcialmente en su dominación, subordinación y discriminación, impidiendo su pleno desarrollo (Naciones Unidas, 1993).

Abordar los derechos humanos de las mujeres -especialmente el derecho a vivir una vida libre de violencia- exige generar estudios científicos interdisciplinarios que aporten información, con materiales de consulta, análisis puntuales válidos, que permitan comprender la realidad y contrastarla para fomentar una transición hacia la reducción o incluso erradicación de la violencia ejercida contra las mujeres (Espinoza, 2019; Gallegos \& Rosales, 2012; Lara, 2013), a partir del desarrollo de estrategias efectivas de prevención, tratamiento y sanción (Cepal, 2010). También hay 
que profundizar en las manifestaciones de la violencia - del tipo desaparición forzada de mujeres- con los objetivos de prevenir el feminicidio (Bejarano, 2014) y generar un conjunto de representaciones que faciliten a la población identificar y reconocer incluso las formas más sutiles de violencia, para con ello inhibir la reproducción de esas conductas (Segato, 2003).

La literatura académica disponible refleja la existencia de diversos estudios realizados que abordan el fenómeno de la violencia ejercida contra las mujeres, del tipo desaparición forzada y feminicidio en el contexto del estado de Chihuahua, (Amnistía Internacional, 2003; Aragón, 2017; Dena, 2018; Lagarde, 2005; Meyen et al., 2010; Monárrez, 2009; Ravelo, 2008), pero no se identificaron trabajos previos con las características y atributos que muestra el presente documento, el cual parte de un sustento epistemológico crítico, bajo un enfoque cualitativo, de tipo exploratorio, no experimental y descriptivo, aplicando una técnica analítica con base en artículos científicos, libros, revistas, normatividad, sitios web, páginas oficiales de organismos nacionales e internacionales, etcétera. Está orientado a identificar los esquemas y mecanismos oficiales existentes, desde los cuales se aborda la observancia de la violencia contra las mujeres, del tipo desaparición forzada y feminicidios en el estado de Chihuahua, abonando con ello al avance del conocimiento relativo, que coadyuve al desarrollo de estrategias efectivas de prevención, reducción, sanción y erradicación de dichas violaciones a los derechos humanos.

\section{VIOLENCIA CONTRA LAS MUJERES}

Abordar la violencia ejercida contra las mujeres, exige comprender previamente la dificultad existente para establecer un concepto de violencia unívoco y satisfactorio que abarque sus distintas manifestaciones, como resultado de procesos complejos (Blair, 2009), incluso se afirmó que no existe una sola teoría capaz de explicar todas las formas de violencia (Semelin, 1983); sin embargo, destacan distintas aportaciones donde cobra relevancia la definición 
emitida por la Organización Mundial de la Salud, misma que define:

La violencia es el uso intencional de la fuerza física, amenazas contra uno mismo, otra persona, un grupo o una comunidad que tiene como consecuencia o es muy probable que tenga como consecuencia un traumatismo, daños psicológicos, problemas de desarrollo o la muerte (Organización Mundial de la Salud, 2020: 1).

Todo acto de violencia se encuentra estrechamente vinculado a una serie de necesidades sociales y/o personales derivadas de limitaciones, y está asociado con un conjunto de carencias personales y sociales originadas por una estructuración deficiente en las distintas oportunidades de contexto. Incide negativamente en la adaptación de los individuos en su entorno social, por lo que resulta útil aproximarse al análisis de la violencia desde la vinculación de tres tipos puntuales de esta: directa, estructural y cultural (Espinoza, 2019), cobrando relevancia identificar cada una de ellas.

Si bien en la violencia directa se identifica con claridad tanto a la víctima como al agresor, en la violencia estructural esto difiere totalmente, ya que esta es invisible y es causada por los procesos de estructuración social, a partir de conflictos existentes entre dos o más grupos de la sociedad en torno a los esquemas de acceso, distribución o posibilidad de uso de recursos y que sistemáticamente se ha mantenido resuelto a favor de un grupo o clase específica, obteniendo mayores beneficios y afectando con ello al resto; por otra parte, la violencia cultural es representativa de la sociedad cuyos simbolismos trascienden a través del tiempo, estos se encuentran presentes en el lenguaje, el arte, la ciencia empírica, la religión, la ciencia formal, entre otros entornos, y es utilizada para legitimar o justificar la violencia, de ahí que se afirme que tanto la violencia directa como la estructural surgen de la violencia cultural, haciendo que estas se perciban como válidas, no equivocadas o cargadas de razón, incluso legitimándolas desde su interiorización y normalización (Galtung, 2016). 
La estructura social conformada por individuos, familias, escuelas, sociedad, espacios laborales, leyes, religiones, etc., asigna roles, características y espacios específicos para hombres y para mujeres, estableciendo sanciones discriminatorias y violentas para quienes no las cumplen, concediendo un papel de superioridad a los hombres, eje central de la violencia ejercida contra las mujeres con la finalidad de mantenerlas en una posición de subordinación y dominación (Espinoza, 2019; Loeza, 2017), de ahí que esta violencia estructural se manifieste al amparo de la violencia cultural que comúnmente presenta la realidad con relativismo o caracteres difusos para evitar que se perciba como acto violento o que se justifiquen, legitimen y normalicen, como puede ser el asesinato por la patria, como algo correcto, en tanto que el asesinato en beneficio propio se considera incorrecto (Galtung, 2016).

A lo largo de la historia tanto a hombres como a mujeres se les ha asignado que desempeñen roles específicos que fungirán como normas de funcionamiento social y estructura de identidad para los sujetos; dichas ideas actualmente se reconocen como estereotipos, los cuales indican cómo deben comportarse las personas de acuerdo a su sexo, raza, grupo social o religión, estableciéndose diferencias socioculturales, avalando y sustentando la violencia contra las mujeres, lo cual constituye una ofensa contra la dignidad humana y una manifestación retrógrada al reproducir relaciones de poder desigual entre hombres y mujeres (Alberdi \& Matas, 2002).

Considerando la relevancia del tema, es necesario precisar que en la literatura académica se aprecia el uso del término violencia de género y violencia contra las mujeres y niñas de forma indistinta, sin embargo, el primero alude a la agresión que se ejerce contra una persona debido a su género dadas las expectativas de cumplimiento de los roles que le son asignados por la sociedad a partir de la cultura en la que están insertos, haciendo por tanto hincapié en la dimensión de género, que puede expresarse de diversas maneras, ámbitos y relaciones como la violencia en el hogar, la violación, el hostigamiento sexual o la muerte, que no es privativo de mujeres y niñas, ya que hombres y niños también 
pueden ser víctimas de la violencia de género, especialmente de violencia sexual. En cuanto al término de violencia contra las mujeres -y niñas - puede incluir varias manifestaciones de violencia derivadas de otras formas de discriminación distintas a la desigualdad de género, como su origen étnico (ONU Mujeres, 2020).

Si bien para algunas mujeres la violencia directa ejercida contra ellas puede ser un acontecimiento eventual, para muchas otras es una situación tan frecuente que incluso es parte de su forma de vida. Esta violencia se manifiesta de distintas formas, tales como humillaciones, desprecio, acoso, descalificación, maltrato, desaparición forzada y la muerte, por señalar algunas; sin embargo, la forma de violencia más perjudicial para las mujeres es la violencia estructural, práctica aprendida en la interacción social que se encuentra normalizada (Magallón, 2005). Como parte del proceso histórico y de una construcción social, a las mujeres se les ha asignado la función principal en la procreación, cuidado de los hijos y del hogar, relegadas al ámbito doméstico, desde la subordinación y no como creadoras. Posteriormente, con el surgimiento de las clases sociales, se les considera objetos de placer para los hombres. Estos roles prevalecen hasta la actualidad (Herrera, 2000) y constituyen violencia ejercida contra las mujeres, ya que al ser estructural, trasciende niveles socioeconómicos y educativos (Red TDT, 2017). Esta violencia es el mecanismo empleado para puntualizar que el poder es un ejercicio masculino sobre el comportamiento, la subjetividad, el cuerpo y los derechos de las mujeres (Zúñiga et al., 2012).

Los costos sociales y económicos de la violencia contra las mujeres son muy graves (Monárrez, 2000), implican un comportamiento aprendido en ámbitos de desigualdad, donde no se reconocen los derechos ni la dignidad de las mujeres y que puede manifestarse en ámbitos laborales, políticos y sociales (Red TDT, 2017); es ejercida por los hombres como mecanismo de control eficiente contra las mujeres para evitar que transgredan el orden social, manteniendo su subordinación ante el poder masculino, cuyas máximas expresiones son la desaparición forzada y el ase- 
sinato de mujeres -feminicidio- (Amorós, 1989; Bejarano, 2014; Monárrez, 2000).

Perspectivas complementarias (Esteves, 2017) indican que la desaparición forzada y la masacre - de mujeres y niñas - constituyen técnicas principales del necropoder, aplicadas ante el ambiente de impunidad que impera. La primera de ellas se emplea para féminas que van de 0 a 30 años, con la finalidad de usar sus cuerpos para someterlos a esclavitud sexual principalmente; en tanto que el feminicidio se usa cuando se rebelan contra la dominación masculina y se ejerce en mujeres de más de 30 años, cuando sus cuerpos ya no tienen utilidad comercial.

Es importante señalar que de acuerdo con la Convención Internacional Para la Protección de Todas las Personas Contra las Desapariciones Forzadas (2006), se considera desaparición forzada:

el arresto, la detención, el secuestro o cualquier otra forma de privación de libertad que sean obra de agentes del Estado o por personas o grupos de personas que actúan con la autorización, el apoyo o la aquiescencia del Estado, seguida de la negativa a reconocer dicha privación de libertad o del ocultamiento de la suerte o el paradero de la persona desaparecida, sustrayéndola a la protección de la ley (Naciones Unidas Derechos Humanos Oficina del Alto Comisionado, 2006).

Por otra parte, una elaborada construcción teórica plantea asignar la categoría del feminicidio al asesinato misógino de mujeres perpetrado por hombres, por el hecho de ser mujeres (Radford \& Rusell, 1992), incluyendo todas las formas de asesinato sexista (Rusell, 2005), lo que implica la destrucción literal de la vida de mujeres y niñas como el máximo acto de sexismo masculino a consecuencia de la posición de discriminación estructural que la sociedad patriarcal atribuye a los roles femeninos, a partir la violación sistemática y reiterada de los derechos humanos de las mujeres (Lagarde, 2005). Desde una perspectiva de género se considera que toda violencia que cause la muerte de la mujer es un feminicidio (Monárrez, 2004), donde independientemente del tipo 
de feminicidio (racista, en masa, íntimo, sexual, etc.), se materializa el uso de un derecho patriarcal que ha prevalecido a través del tiempo y que somete el cuerpo de las mujeres (Dworkin,1997). Por tanto, la violencia ejercida contra las mujeres constituye una violación a los derechos humanos (Galtung, 2016), donde la desaparición forzada y el feminicidio son considerados crímenes de lesa humanidad (Carbajal et al., 2019).

\section{LOS DERECHOS HUMANOS}

Los derechos humanos son determinantes para el desarrollo de la sociedad (Castilla, 2015), esenciales para mantener el orden y la paz mundial (Douzinas, 2006; Falk, 2002) y deben contribuir a la felicidad y bienestar de la sociedad (Calvo, 2016). Dada su relevancia es importante conocer su fundamentación teórica, donde destacan dos corrientes de pensamiento: el Iusnaturalismo y el Iuspositivismo (Carbonell, 2013); la primera afirma la existencia de un orden suprapositivo, inviolable y universal, que contiene los valores de todo ordenamiento (Aguilera, 2007). En esa misma línea surgen algunos trabajos (López, 2009; Maldonado, 2010) que refieren la existencia de diversos tipos de iusnaturalismo: ontológico, radical, medieval o dogmático y afirman que los derechos humanos son otorgados por Dios a los hombres, bajo valores y principios inmutables, incuestionables, universales y eternos. Posteriormente, desde un proceso de racionalización y secularización aparecen el iusnaturalismo racionalista, moderno crítico, mecanicista, deontológico o moderado, afirmando que los derecho humanos son inherentes al hombre por su naturaleza, por el hecho de existir y de ser personas (Amnistía Internacional, 2018).

Por su parte, el Positivismo o Iuspositivismo sostiene que los derechos - humanos - solamente existirán en la medida en que se encuentren contenidos en un ordenamiento jurídico, donde las normas vigentes constituyen la única fuente del derecho (Amnistía Internacional, 2018; Carbonell, 2013; Maldonado, 2010). Tales postulados constituyen el fundamento de ideologías como el utilitarismo y el positivismo jurídico, y aunque ambas se orientan a 
procurar el mayor bien para el mayor número de personas (Álvarez, 2015; López, 2009), este último plantea especialmente que los derechos humanos forman parte de los esquemas normativos de los Estados, por lo que se integran al ámbito internacional (Calvo, 2016; Ferrajoli, 2006; Polo, 2000), sustentándose desde ahí el presente documento.

Independientemente de las distintas perspectivas desde las cuales se han abordado los derechos humanos, estos se sustentan en los principios de igualdad y libertad de las personas y se traducen en obligaciones positivas, que consisten en realizar acciones para su desarrollo, protección y promoción, o deberes negativos, que consisten en omitir, no hacer, y/o abstenerse de tomar acciones que afecten o lesionen tales derechos (Garzón, 1986; Nelson, 1981) relativos a todos aquellos bienes que constituyen la dignidad humana, conocidos como bienes primarios (Laporta, 1987), y que bajo el marco de conceptos jurídicos se denominarán derechos subjetivos (Ferrajoli, 2006), y que constituyen el lenguaje hegemónico de la dignidad humana (De Sousa, 2014) y de su afirmación frente al Estado, orientado a que cada individuo tenga acceso a los recursos necesarios para la realización de sus planes de vida (Carpizo, 2011; Nikken, 1994; Williams, 2002).

A partir de la Declaración Universal de los Derechos Humanos adoptada por la Asamblea General de las Naciones Unidas en 1948, cobra relevancia la conceptualización de los mismos, robusteciéndose en las últimas décadas. Se identifican en la literatura distintas aproximaciones donde, dada su complejidad, se configuran a partir de la corriente teórica o naturaleza a la que se circunscriba, de tal forma que si bien no existe uniformidad de criterios que permitan definir el concepto de Derechos Humanos (Polo, 2000), de las distintas aportaciones plasmadas en los apartados superiores es posible considerar que los derechos humanos constituyen aquellas condiciones inherentes al ser humano necesarias para garantizar el respeto a su dignidad, que permitan su desarrollo y trascendencia, ya sea de forma individual, social y/o colectiva, en todo tiempo, lugar y circunstancia. 
Así, desde los principios de igualdad y libertad de las personas, los derechos humanos se traducen en obligaciones o deberes (Garzón, 1986; Nelson, 1981), que pueden ser positivas a partir de las cuales se exige la realización de acciones orientadas a proteger y promover dichos derechos, o bien en obligaciones negativas, consistentes en abstenerse u omitir acciones que violenten la dignidad humana (Garzón, 1986). Sin embargo, la noción de derechos humanos alude al deber internacional de respetar la dignidad, la libertad y la seguridad de las personas, sin discriminación y en condiciones de igualdad universal — quienes nacen libres siendo simplemente seres humanos-, pero múltiples factores, tanto físicos como sociales generan diferencias entre ellas, de ahí que el derecho constituye la herramienta legal desde la cual se aspira a igualarlos (Pinto, 2017). El Derecho Internacional de los Derechos Humanos plantea la obligatoriedad que los Estados asumen de respetar, proteger y garantizar los derechos humanos (Naciones Unidas, Derechos Humanos, Oficina del Alto Comisionado, 2019).

\section{PERSPECTIVA INTERNACIONAL SOBRE LA VIOLENCIA CONTRA LAS MUJERES}

Es importante mencionar que los propósitos de la comunidad internacional, planteados a través de la Organización de las $\mathrm{Na-}$ ciones Unidas, giran en torno a preservar la paz y la seguridad mundial, desplegando una serie de medidas colectivas orientadas a prevenir y eliminar amenazas a la paz, fomentando relaciones de respeto y de amistad entre las naciones, la solución pacífica de los conflictos sociales, económicos, culturales o humanitarios, basados en el respeto a los derechos humanos sin hacer distinción por motivos de raza, sexo, idioma o religión, sirviendo de eje armonizador de los esfuerzos internacionales (Naciones Unidas, Derechos Humanos, Oficina del Alto Comisionado, 2019).

Las Naciones Unidas afirman que la violencia contra la mujer constituye una violación de sus derechos humanos, se traduce total o parcialmente en dominación, subordinación y discriminación respecto de los hombres, impidiendo su pleno desarrollo (Nacio- 
nes Unidas, 1993). Además, los derechos humanos de las mujeres comúnmente son limitados, cuestionados y/o negados, tanto en contextos locales como globales (Faci \& Victory, 2017). Se destaca que grupos como indígenas, migrantes, niñas, fetos hembras, adolescentes y ancianas, por señalar algunos, son particularmente más vulnerables a la violencia (Andrés, 2004).

En la Conferencia Internacional Sobre Desarrollo Social de la Mujer, celebrada en Copenhague en 1995, se enfatizó en la necesidad de avanzar en esquemas de igualdad y empoderamiento de las mujeres y la eliminación de todas las formas de violencia contra ellas, cuyas máximas manifestaciones son la desaparición forzada y el feminicidio. También se exhortó a los gobiernos a tomar medidas puntuales de prevención y eliminación de todas las formas de explotación, maltrato, acoso y violencia contra mujeres, niños, niñas y adolescentes, incluyendo esquemas de rehabilitación para las víctimas (Naciones Unidas, 1995).

En las últimas décadas la violencia contra las mujeres cobra especial relevancia y destaca su pertinencia en la agenda política de la comunidad internacional, generando convenciones, declaraciones y protocolos regionales de atención, que se han ido adaptando a cada contexto en concordancia con los tratados internacionales de derechos humanos (Segato, 2004). En 1993 la Organización de las Naciones Unidas proclamó la Declaración Sobre la Eliminación de la Violencia Contra la Mujer (Naciones Unidas, 1993), en la cual se reconoce la urgente necesidad de aplicar a la mujer en condiciones de igualdad el goce y la protección de todos y cada uno de los derechos y principios contemplados en: a) la Declaración Universal de los Derechos Humanos, b) el Pacto Internacional de Derechos Civiles y Políticos, c) el Pacto Internacional de Derechos Económicos, Sociales y Culturales, d) la Convención sobre la eliminación de todas las formas de discriminación contra la mujer, y e) la Convención contra la Tortura y Otros Tratos o Penas Crueles, Inhumanos o Degradantes. Asimismo, se afirma que toda violencia ejercida contra la mujer constituye una violación a sus derechos humanos y le impone una situación de subordinación respecto del hombre, por lo que la Conferencia Mundial de 
Derechos Humanos (1993) afirma que las violencias por razón de sexo son el resultado de prejuicios culturales que son incompatibles con la dignidad y el valor de la persona, por lo cual deben ser eliminadas (Naciones Unidas, Oficina del Alto Comisionado, 1993). Destaca que los artículos 1 y 2 de la Declaración Sobre la Eliminación de la Violencia Contra la Mujer, de 1993, establecen que:

...por "violencia contra la mujer" se entiende todo acto de violencia basado en la pertenencia al sexo femenino que tenga o pueda tener como resultado un daño o sufrimiento físico, sexual o sicológico para la mujer, así como las amenazas de tales actos, la coacción o la privación arbitraria de la libertad, tanto si se producen en la vida pública como en la vida privada... Se entenderá que la violencia contra la mujer abarca los siguientes actos, aunque sin limitarse a ellos: La violencia física, sexual y sicológica que se produzca en la familia, incluidos los malos tratos, el abuso sexual de las niñas en el hogar, la violencia relacionada con la dote, la violación por el marido, la mutilación genital femenina y otras prácticas tradicionales nocivas para la mujer, los actos de violencia perpetrados por otros miembros de la familia y la violencia relacionada con la explotación; la violencia física, sexual y sicológica perpetrada dentro de la comunidad en general, inclusive la violación, el abuso sexual, el acoso y la intimidación sexuales en el trabajo, en instituciones educacionales y en otros lugares, la trata de mujeres y la prostitución forzada y aquella perpetrada o tolerada por el estado donde esta ocurra (Naciones Unidas Derechos Humanos Oficina del Alto Comisionado, 1993: 2).

En 1994 la comunidad internacional realizó distintos esfuerzos orientados a abonar a la erradicación de la violencia ejercida contra las mujeres, de ahí que el Comité de Derechos Humanos instruyó a los gobiernos a abstenerse de cometer actos violentos contra las mujeres -y niñas-, además de prevenirlos, investigarlos y castigarlos. Por otra parte, en ese mismo año se celebra en El Cairo la Conferencia Internacional Sobre Población y Desarrollo, donde se reafirma la necesidad de avanzar en esquemas de igual- 
dad y empoderamiento y la eliminación de todas las formas de violencia contra la mujer.

En América se celebra la Convención interamericana para prevenir, sancionar y erradicar la violencia contra la mujer "Convención Belem do Pará” (1994), en la cual se reconoce que todas las formas de violencia, ya sean física, psicológica, sexual y económica contra las mujeres constituyen una ofensa a la dignidad humana y evidencian disimiles condiciones en las relaciones de poder entre hombres y mujeres a lo largo de la historia y que han conducido a su dominación y discriminación al forzarlas a una situación de subordinación respecto del hombre, lo que implica una violación a los derechos humanos, por lo cual se enfatiza el derecho de las mujeres a vivir una vida libre de violencia y discriminación, ejerciendo libre y plenamente todos sus derechos (Organización de Estados Americanos, 1994). Un año más tarde, en la Cumbre Mundial para el Desarrollo Social, se condenan categóricamente la violencia y la violación en el hogar contra mujeres, niños y niñas, generando un programa de acción orientado a su prevención (Naciones Unidas, 1995). Luego, en la Cuarta Conferencia Mundial sobre la Mujer, de Beijing, se establece que la eliminación de la violencia contra las mujeres es esencial para la igualdad, el desarrollo y la paz y se exhorta a los Estados a reconocer la vulnerabilidad de las mujeres -y niñas - frente a la violencia (ONU Mujeres, 2016; Organización de Estados Americanos, 2017).

Los primeros instrumentos jurídicos internacionales que reconocen los principios de igualdad y no discriminación entre los seres humanos -entre hombres y mujeres - son: a) la Carta de las Naciones Unidas firmada en 1945 y b) la Declaración Universal de los Derechos Humanos, promulgada en 1948 bajo la tutela de la Organización de las Naciones Unidas (Naciones Unidas, Derechos Humanos, Oficina del Alto Comisionado, 2019). Así, a partir de la segunda mitad del siglo XX se identifican distintos esfuerzos internacionales por generar mecanismos de observancia de los derechos humanos, destacando la creación de la Comisión de la Condición Jurídica y Social de la Mujer. Además, en 1967 se emite 
la Declaración sobre la eliminación de la discriminación contra la mujer (ONU Mujeres, 2019).

Entre las acciones realizadas desde la Organización de las Naciones Unidas, orientadas a erradicar la discriminación hacia las mujeres en el mundo, destacan: la designación de 1975 como el Año Internacional de la Mujer; la declaración del periodo comprendido entre 1975 y 1985 como el Decenio de las Naciones Unidas para la Mujer (ONU Mujeres, 2019); la creación en 1976 de UNIFEM, Fondo de las Naciones Unidas para la mujer, orientado a proveer asistencia técnica y financiera a iniciativas que fomenten el empoderamiento y cuyos objetivos buscan erradicar la violencia y todas las formas de discriminación que padecen las mujeres en todo el planeta (ONU Mujeres, 2011). También destacan cuatro conferencias mundiales sobre los derechos de las mujeres, celebradas en México (1975), Copenhague (1980), Nairobi (1985) y Beijing (1995). En la primera de ellas (1975) destaca la participación de 133 delegaciones de distintos Estados, de las cuales 113 eran representadas por mujeres, tomando así un papel protagónico en la definición de aquellas pautas de acción que debían observar los gobiernos de la comunidad internacional para garantizar a las mujeres igualdad de acceso a la educación, la alimentación, la salud, la planificación familiar, la vivienda, el trabajo y su participación política (ONU Mujeres, 2016).

\section{INSTRUMENTOS INTERNACIONALES DE OBSERVANCIA DE LOS DERECHOS HUMANOS DE LAS MUJERES}

Para 1979, la Asamblea General de Naciones Unidas adopta la Convención sobre la eliminación de todas las formas de discriminación contra la mujer (CEDAW), instrumento internacional que puntualiza la discriminación contra las mujeres y plantea las directrices para su erradicación. Los 165 Estados que la han ratificado se obligan a adoptarlas, a sentar las bases legales necesarias para que exista igualdad formal entre hombres y mujeres. Llevan a cabo una serie de medidas internas para eliminar las violaciones de derechos humanos contra las mujeres, orientadas a lograr la 
igualdad sustantiva (igualdad de trato, oportunidades y ejercicio pleno de los derechos humanos para hombres y mujeres), además de comprometerse a presentar un informe tetra anual en el que evalúen la situación relativa (Observatorio Ciudadano de los Derechos de las Mujeres, 2019; ONU Mujeres México, 1979).

La segunda Conferencia Mundial sobre la Mujer, celebrada en Copenhague (1980), giró en torno a la Comisión de la Condición Jurídica y Social de la Mujer, y se reflexionó sobre los resultados de la primera Conferencia Mundial. Se planteó pasar del simple reconocimiento jurídico a la consecución efectiva del derecho de igualdad entre hombres y mujeres. Se determinó buscar las causas de la falta de observancia, refiriéndose a la poca participación de los hombres, insuficiente voluntad política de los gobiernos, limitada participación de mujeres en puestos de toma de decisiones, reducidos servicios sociales de apoyo a las mujeres, poco reconocimiento de las mujeres en la sociedad, escaso acceso a servicios de financiamiento y poca sensibilización entre las propias mujeres. Se propuso abordar tal problemática a partir de tres temas fundamentales: a) la igualdad en el acceso a la educación, b) la igualdad de oportunidades en el empleo, y c) la atención a la salud de las mujeres (Coordinadora de la Mujer, 1980).

La tercera Conferencia Mundial sobre la Mujer, celebrada en Nairobi (1985), contó con la participación de 157 Estados y se abordó la temática desde una nueva perspectiva al reconocer la necesidad de la sociedad de contar con la participación de las mujeres en todos los ámbitos de la vida, y no solo como receptoras de derechos, por lo que se llama a los gobiernos a implementar medidas de carácter jurídico que permitan alcanzar la igualdad entre hombres y mujeres en el ámbito laboral y en la participación social y política, incluyendo en espacios de toma de decisiones (United Nations Women, 2019).

Desde los esfuerzos internacionales previamente referidos es posible abordar el presente tema, considerando que la igualdad jurídica es tan solo una parte de la igualdad real o de facto, a la que las Naciones Unidas llaman igualdad sustantiva, y que fomenta una mayor participación de las mujeres en todos los ámbitos de la 
sociedad. Pero será hasta la cuarta Conferencia Mundial sobre la Mujer, celebrada en Beijing, 1995, cuando se integra el concepto de género y su transversalidad, mismo que desde una perspectiva social, cultural e histórica, contempla las relaciones entre hombres y mujeres, tomando en cuenta los roles atribuidos socialmente a cada uno de ellos. En la Declaración aprobada por unanimidad por los 189 Estados participantes destacan, entre otros temas, el reconocimiento de la propia diversidad existente entre las mujeres, a partir de las diferentes circunstancias que experimentan y en las que se desarrollan; la ardua labor de aquellas mujeres que trabajaron por visibilizar la importancia y problemática propia, afirmando que los derechos de las mujeres -y de las niñas - son derechos humanos; la participación de las mujeres en todos los ámbitos de la sociedad como una condición fundamental para alcanzar la igualdad, el desarrollo, la paz y la construcción de un mundo mejor; el énfasis en la importancia de prevenir y eliminar todas las formas de violencia contra las mujeres y las niñas (ONU Mujeres, 2016).

Buscando lograr la igualdad - sustantiva - y la eliminación de la discriminación por motivos de género, fomentar la participación de las mujeres en el desarrollo, y lograr una mayor contribución de las mujeres a la paz mundial, se establecieron doce áreas críticas a resolver: la mujer y la pobreza, educación y capacitación de la mujer, la salud de la mujer, la violencia ejercida contra la mujer, la mujer y los conflictos armados, la mujer y la economía, la mujer en el ejercicio del poder, la adopción de decisiones, mecanismos institucionales para el adelanto de la mujer, los derechos humanos de la mujer, la mujer y los medios de comunicación y difusión, la mujer y el medio ambiente (United Nations Women, 2019).

Cabe mencionar que la Asamblea General decidió efectuar evaluaciones sobre la observancia de los acuerdos de Beijing cada cinco años. El evento de evaluación denominado "La mujer en el año 2000: igualdad entre los géneros, desarrollo y paz para el siglo XXI" se llevó a cabo en Nueva York, y se emitieron una declaración política y nuevas medidas e iniciativas para la aplicación del Plan de Acción de Beijing (Naciones Unidas, 2000). La evaluación 
correspondiente al año 2005 se realizó en el marco de la Comisión de la Condición Jurídica y Social de la Mujer, la relativa al año 2010 y la evaluación de 2015 fue denominada Beijing +20 (Naciones Unidas, Derechos Humanos, Oficina del Alto Comisionado, 2019).

\section{ESQUEMAS DE PROTECCIÓN EN MÉXICO FRENTE A LA VIOLENCIA CONTRA LAS MUJERES}

En respuesta a los tratados internacionales ratificados de los cuales México forma parte, se generaron esquemas de protección en materia de derechos humanos, sin embargo, es hasta 2011 cuando el gobierno mexicano los eleva a nivel constitucional y establece la obligatoriedad de reconocer, respetar, promover y generar mecanismos tanto de protección como de defensa de tales derechos (Cámara de Diputados, 2019). Se incluye a los contenidos en convenios y tratados internacionales de los cuales México forma parte, con excepción de aquellos para los cuales se haya emitido reserva (Carbonell, 2012; CNDH, 2018; Suprema Corte de Justicia de la Nación, Universidad Nacional Autónoma de México y Fundación Konrad Adenauer, 2013). También se creó la Comisión Nacional de los Derechos Humanos, institución oficial encargada de estudiar, promover, divulgar y defender la observancia de tales derechos (Comisión Nacional de los Derechos Humanos, 2018) y se expidió la Ley General de Acceso de las Mujeres a una Vida Libre de Violencia, orientada a establecer la coordinación entre la federación, las entidades federativas, la Ciudad de México y los municipios, para prevenir, investigar, sancionar, reparar y erradicar la violencia contra las mujeres (Cámara de Diputados, 2019).

Así, ante la necesidad de contrarrestar los obstáculos que las mujeres enfrentan en el ejercicio de sus derechos humanos, y para cumplir con los requerimientos planteados desde el ámbito federal, se ha estructurado un marco normativo orientado a enfrentar dichas condiciones y que, de acuerdo con la Cámara de Diputados (2011) y al Instituto Nacional de las Mujeres (2007; 2019), destacan: 
- Ley Federal para Prevenir y Erradicar la Discriminación

- Ley General para la Igualdad entre Mujeres y Hombres

- Ley General de Acceso de las Mujeres a una Vida Libre de Violencia

- Reglamento de la Ley General de Acceso de las Mujeres a una Vida Libre de Violencia (2008).

En 2012 se emite el decreto a través del cual se reforman y adicionan las disposiciones del Código Penal Federal, de la Ley General de Acceso de las Mujeres a una Vida Libre de Violencia, de la Ley Orgánica de Administración Pública Federal y de la Ley Orgánica de la Procuraduría General de la República (Cámara de Diputados del H. Congreso de la Unión, 2018).

Es importante destacar que el Secretariado Ejecutivo del Sistema Nacional de Seguridad Pública (2019) además de presentar las cifras de incidencia delictiva, tanto del fuero común como del federal, ha desarrollado un reporte estadístico de información sobre violencia contra las mujeres, que se complementa con información sobre las llamadas de emergencia realizadas al número único 911; además se instruye a las legislaturas estatales para que desde su competencia realicen las reformas necesarias (Cámara de Diputados del H. Congreso de la Unión, 2019; Instituto Nacional de las Mujeres, 2019), por lo cual todas las entidades federativas cuentan con sus propias normas y en algunos casos con comisiones legislativas locales orientadas a la atención de la violencia contra la mujer.

\section{MECANISMOS DE ACCESO A LA JUSTICIA EN MATERIA DE DERECHOS HUMANOS EN EL ESTADO DE CHIHUAHUA}

Dada la obligación constitucional y convencional del gobierno mexicano de garantizar a las mujeres la igualdad sustantiva y el pleno goce de todos sus derechos en todo el territorio nacional, se apoya para ello en las entidades federativas y se generan distintos mecanismos para su observancia (Cámara de Diputados del H. Congreso de la Unión, 2018; Comisión Nacional de los Derechos 
Humanos México, 2018). Así, en el estado de Chihuahua se reconocen tales compromisos y se adoptan de manera conjunta, ya que debido a los antecedentes existentes respecto a la violencia ejercida contra las mujeres, se considera un tema prioritario. Se reconoce que las mujeres siguen siendo objeto de actos de violencia y discriminación, sujetas a distintas violaciones a sus derechos humanos y dignidad, lo que se traduce en obstáculos para que alcancen un nivel de bienestar, pleno desarrollo y autonomía (Consejería y Consultoría Psicojurídica, S. C., 2010).

Desde esa realidad se generan distintos mecanismos y políticas públicas bajo los principio de igualdad y no discriminación, orientados a garantizar el goce de los derechos económicos, sociales, culturales, civiles y políticos de las mujeres. Se operacionalizan como reformas legales en la entidad, tales como: la Reforma al Código Civil para el Estado de Chihuahua, al Código de Procedimientos Civiles para el Estado de Chihuahua, la migración hacia un Sistema de Justicia Penal Acusatorio bajo un esquema de Juicios Orales y la Reforma al Código de Procedimientos Penales para el Estado de Chihuahua, que establece penas más severas para los delitos relativos a la violencia contra las mujeres, principalmente los feminicidios.

Además, de acuerdo con el Congreso del Estado de Chihuahua (2018), en la entidad se promulgaron distintos instrumentos normativos, tales como:

- Ley Estatal del Derecho de las Mujeres a una Vida Libre de Violencia

- Ley de Igualdad entre Mujeres y Hombres del Estado de Chihuahua

- Ley de atención a víctimas u ofendidos del delito del Estado de Chihuahua

- Ley de Justicia Penal Alternativa para el Estado de Chihuahua

También se instauran el Comité para la Eliminación de la Discriminación contra la Mujer y la Unidad de Igualdad de Género 
en el Congreso del Estado (Congreso del Estado de Chihuahua, 2019). Destacan la creación del Instituto Chihuahuense de las Mujeres, organismo enfocado en generar esquemas de prevención de la violencia contra las mujeres, sensibilización, capacitación, etcétera; y principalmente la Fiscalía Especializada en Atención a Mujeres Víctimas del Delito por Razones de Género, orientada a investigar de manera especializada los delitos cometidos en contra de mujeres y niñas.

Sin embargo, las cifras logran evidenciar que no obstante los distintos esfuerzos realizados, las niñas y mujeres siguen siendo objeto de actos de violencia y discriminación, lo que viola los principios de igualdad sustantiva y del respeto de su dignidad humana. Constituyen un grupo en situación de vulnerabilidad, condición que facilita que el fenómeno de la violencia se presente con mayor magnitud. Esto lleva a las mujeres a experimentar desigualdad, discriminación e intolerancia, dificultando su participación en las mismas condiciones que el hombre en la vida política, social, cultural y económica, y entorpece con ello su desarrollo para que alcancen su plena autonomía al comprometer el ejercicio y goce de sus derechos y el acceso a los sistemas de justicia (Lara, 2013; Martínez \& Elizondo, 2018).

\section{CONCLUSIONES}

Desde las aportaciones de Galtung (2016) es posible concluir que la violencia ejercida contra las mujeres -en el Estado de Chihuahua- obedece a la combinación de tres tipos específicos: la violencia directa y la estructural, al amparo de la violencia cultural que la ha justificado, legitimado y normalizado. No obstante, la mayoría de los esfuerzos oficiales identificados giran en torno a esquemas normativos, al considerar que la violencia contra las mujeres puede solucionarse incrementando las penas o tipificando nuevos delitos y asignando la correspondiente sanción, buscando que los individuos sean intimidados por agravar las penas y dejen de cometer delitos. 
Es importante señalar que lo anterior constituye una problemática multifactorial que no puede solucionarse únicamente a través del sistema penal, sino que requiere transitar de políticas criminalizadoras a políticas criminológicas (Espinoza, 2019), que deriven en modificaciones profundas en el aspecto cultural y trasciendan a la estructura social, consideradas origen de la violencia contra las mujeres, por lo que es fundamental enfatizar en el fortalecimiento y desarrollo de nuevas estrategias efectivas y eficientes de prevención.

La violencia contra las mujeres constituye una violación a los derechos humanos, es un atentando contra la dignidad de las personas, reproduce los esquemas de relaciones de poder desigual entre hombres y mujeres, lo que retrasa el desarrollo de estas últimas. La violencia se considera como uno de los principales problemas sociales a nivel mundial y es una de las principales preocupaciones de la comunidad internacional, misma que ha desplegado una serie de instrumentos de protección hacia las mujeres, orientados a erradicarla, destacando las distintas convenciones y tratados internacionales que inciden directamente en la normatividad de aquellos Estados que los ratifican.

Partiendo del compromiso legítimo del gobierno mexicano con la sociedad y de los compromisos internacionales adquiridos, el marco jurídico en México presenta avances muy importantes en materia de derechos humanos, cuyos cambios se basan en los principios de igualdad, libertad y no discriminación, considerando que los seres humanos nacen libres e iguales en valor y dignidad. Así, en dicho país todas las personas pueden invocar todos los derechos y libertades plasmados en el marco jurídico mexicano, especialmente la igualdad real entre hombres y mujeres, referida como igualdad sustantiva.

Consecuentemente, dichos avances cobran un lugar preponderante en la propia normatividad del estado de Chihuahua y los esquemas de impartición de justicia, al ser la primera entidad federativa a nivel nacional en transitar de un sistema de justicia penal tradicional a uno acusatorio, bajo una reforma penal que in- 
tegra una mayor protección para las mujeres chihuahuenses, estableciendo penas más severas especialmente para los feminicidas.

El presente trabajo identifica los distintos instrumentos internacionales, federales y estatales relativos a la observancia de los derechos humanos de las mujeres, especialmente el derecho a vivir una vida libre de violencia. Aunque es posible observar la existencia de avances significativos en materia de derechos humanos, es necesario señalar que también hay asignaturas pendientes que exigen una reflexión permanente, reconociendo que la violencia contra las mujeres es una realidad en el estado de Chihuahua, ya que las cifras relativas reflejan que las manifestaciones de este tipo de violencia se han incrementado considerablemente en dicha entidad federativa.

Ciertamente, la materialización de los derechos humanos de las mujeres, especialmente el derecho a vivir una vida libre de violencia, en el mundo y en el estado de Chihuahua, exige un compromiso de trabajo integral, sustentado en un marco normativo adecuado. Hay que considerar que la violencia se recrudece cuando las mujeres viven en situación de pobreza, ya que tienen un limitado acceso a la alimentación, salud, educación y oportunidades laborales y de participación social en condiciones de igualdad con los hombres. Esto las posiciona como grupo en situación de vulnerabilidad que históricamente se ha normalizado, dificultando su reconocimiento, denuncia y atención.

El presente trabajo aborda la problemática relativa a la violencia ejercida contra las mujeres del tipo desaparición forzada y feminicidio desde la fundamentación y función de los derechos humanos, por lo que se recomienda para futuras investigaciones profundizar desde una perspectiva de género, ya que las condiciones que favorecen la violencia contra cualquier grupo -incluyendo la ejercida contra las mujeres-, son causadas por ideas y prejuicios sociales, cobrando relevancia la corresponsabilidad social (Lamas, 1996; Pizarro, 2017).

Además es determinante ir a la raíz del fenómeno e identificar aquellas condiciones que hacen a las mujeres proclives de experimentar un estado de vulnerabilidad frente a la violencia 
contra ellas, del tipo desaparición forzada y feminicidio, abonando al conocimiento relativo a las causas, orientado a la erradicación del "exterminio de mujeres" (Monárrez, 2004). Se requiere exigir justicia para quienes han sido asesinadas y quienes permanecen desaparecidas, en la conciencia de que dicha violencia parte de anclajes sociales, estructurales y culturales que deben trastocarse. $\mathrm{Su}$ erradicación requiere no solo acciones correctivas, sino preventivas eficaces, atención pertinente y de calidad y sanciones expeditas. La prevención nunca será eficaz si no se sustenta en los principios de la educación que busquen acompañar y ayudar a la mujer a desarrollar aquellos aspectos que la hacen que se "empodere", tenga conocimiento sobre sí misma, autoestima y autonomía suficiente para tomar las riendas de su vida y cambiar la sumisión, la tutela, la obediencia, el silencio, el sufrimiento, el dolor, el desprecio y la violencia, por la independencia, la toma de decisiones, la negociación, el respeto, la alegría, el placer, la solidaridad y las relaciones de paz.

\section{BIBLIOGRAFÍA}

Aguilera, R. (2007). "Universalidad de los derechos humanos y crítica de las teorías de la naturaleza humana en el prabmatismo de Richard Rorty". Universitas Revista de Filosofía, Derecho y Política, enero(5). Pp. 47-75.

Alanís, M. (2017). Violencia política hacia las mujeres. Respuesta del estado ante la falta de una ley en México. En S. D. Instituto de Investigaciones Jurídicas (Ed.), Cuando hacer política te cuesta la vida. Ciudad de México: Universidad Nacional Autónoma de México.

Alberdi, I., \& Matas, N. (2002). La violencia doméstica. Informe sobre los malos tratos a las mujeres en España. Madrid: Fundación La Caixa.

Álvarez, A. (2015). Problemática de la mujer inmigrante islámica y efectos jurídicos y sociales en el derecho positivo español. Recuperado el 30 de marzo de 2020, de https://sudocument. ulpgc.es/bitstream/10553/25624/4/0738508_00000_0000.pdf 
Amnistía Internacional. (2003). México muertes intolerables: 10 años de desapariciones y asesinatos de mujeres en Ciudad Juárez y Chihuahua (Primera ed.). Madrid: Amnistía Internacional.

Amnistía Internacional. (2018). Historia de los derechos humanos. Recuperado el 30 de marzo de 2020, de Iusnaturalismo y positivismo: http://www.amnistiacatalunya.org/edu/es/historia/ inf-intro3.html

Amorós, C. (1989). Violencia contra las mujeres y pactos patriarcales. En Violencia y sociedad patriarcal. Madrid: Pablo Iglesias. Anderson, J., \& Gerbing, D. (1988). "Structural Equation Modeling in Practice: A Review and Reccomended Two-Step Approach”. Psychological Bulletin, 2(3). Pp. 411-423.

Andrés, P. (2004). Violencia contra las Mujeres. Violencia de género. En La violencia contra las mujeres. prevención y detección. Madrid: Ediciones Días de Santos.

Aragón, L. (2017). Activistas y defensoras de derechos humanos. Recuperado el 18 de abril de 2020, de http://diposit.ub.edu/ dspace/bitstream/2445/128970/1/TFM_Liliana_Arag\%C3\%B3n_ Castro.pdf

BBC News Mundo. (2016). País por país: el mapa que muestra las trágicas cifras de los feminicidios en América Latina. Recuperado el 2 de abril de 2020, de https://www.bbc.com/mundo/ noticias-america-latina-37828573

Bejarano, M. (2014). El feminicidio es solo la punta del iceberg. Región y sociedad, 26(4). Pp. 13-44.

Blair, E. (2009). Aproximación teórica al concepto de violencia: avatares de una definición. Política y cultura (32). Pp. 9-33.

Caballero, J. A. (2011). Derechos de los jóvenes. En H. Fix-Zamudio, \& D. Valadés, Instituciones sociales en el constitucionalismo contemporáneo. México: Universidad Nacional Autónoma de México y El Colegio Nacional.

Calvo, A. (2016). "Iusnaturalismo y positivismo jurídico de los derechos fundamentales de la persona en las condiciones peruanas del S. XX". Educare et Comunicare: Revista de Investigación de la Facultad de Humanidades, 4(1). Pp. 6-14. 
Cámara de Diputados (2011). Ley General de Acceso de las Mujeres a una Vida Libre de Violencia. Recuperado el 9 de junio de 2019, de http://www.cofemer.gob.mx/LGAMVLV.pdf

Cámara de Diputados (2019). Constitución Política de los Estados Unidos Mexicanos. Recuperado el 23 de mayo de 2019, de http://www.diputados.gob.mx/LeyesBiblio/ref/cpeum.htm

Cámara de Diputados del H. Congreso de la Unión (2018). Cámara de Diputados del H. Congreso de la Unión. Obtenido de Constitución Política de los Estados Unidos Mexicanos de 1917: http://www.diputados.gob.mx/LeyesBiblio/pdf/1_270818.pdf

Cámara de Diputados del H. Congreso de la Unión (2019). Cámara de Diputados del H. Congreso de la Unión. Obtenido de Constitución Política de los Estados Unidos Mexicanos de 1917: http://www.diputados.gob.mx/LeyesBiblio/pdf/1_090819.pdf

Carbajal, E., Monárrez, J., \& Medina, R. (2019). Nuevas guerras y crímenes contra la humanidad: las mujeres sin cuerpo y sin espacio. En D. G. Nación (Ed.), Feminismos y Derecho: Un diálogo interdisciplinario en torno a los debates contemporáneos. Ciudad de México: Centro de Estudios Constitucionales de la Suprema Corte de Justicia.

Carbonell, M. (2012). La reforma constitucional en materia de derechos humanos: princiaples novedades. Recuperado el 3 de julio de 2019, de http://www.miguelcarbonell.com/articulos/ novedades.shtml.

---. (2013). Derechos fundamentales y democracia. México, D. F.: Instituto Federal Electoral.

Carpizo, J. (2011). "Los Derechos Humanos: naturaleza, denominación y características". Cuestiones Constitucionales, 25. Pp. 3-29.

Castilla, B. (2015). "En torno a la fundamentación de la dignidad personal”. Foro Nueva Epoca Academia de Número de la Real Academia de Cobroes de España, 18(1). Pp. 61-80.

CEDAW. (1993). Declaración sobre la eliminación de la violencia contra la mujer. Organización de las Naciones Unidas. Recuperado el 05 de enero de 2019, de https://www.ohchr.org/sp/ professionalinterest/pages/violenceagainstwomen.aspx 
CEPAL (Comisión Económica para America Latina y el Caribe) (2010). Estudio de la información sobre la violencia contra la mujer en América Latina y el Caribe. Recuperado el 13 de junio de 2019, de https://repositorio.cepal.org/bitstream/handle/11362/5826/1/S0900880_es.pdf

Comisión Nacional de los Derechos Humanos (2018). Informe anual de actividades 2018. Recuperado el 21 de mayo de 2019, de http://informe.cndh.org.mx/menu.aspx?id=23

---. (2018). Violencia política contra las mujeres en razón de género. Recuperado el 28 de febrero de 2019, de http://www.cndh. org.mx/sites/all/doc/OtrosDocumentos/Doc_2018_056.pdf Congreso del Estado de Chihuahua (2018). Decreto No. LXV/ RFLYC/0854/2018 XVI P.E. Recuperado el 12 de diciembre de 2019, de http://www.congresochihuahua2.gob.mx/biblioteca/ decretos/archivosDecretos/6872.pdf

Congreso del Estado de Chihuahua 2018-2021 (2019). Unidad de Igualdad de Género. Obtenido de https://www.congresochihuahua.gob.mx/unidadGenero/unidad.php

Consejería y Consultoría Psicojurídica, S. C. (2010). Diagnóstico Técnico-Jurídico de los Derechos de las Mujeres en el Estado Libre y Soberano de Chihuahua. Recuperado el 15 de diciembre de 2019, de http://cedoc.inmujeres.gob.mx/ftpg/Chihuahua/Chih03.pdf

Coordinadora de la Mujer (1980). 2da Conferencia Mundial sobre la Mujer Copenhague. Recuperado el 17 de diciembre de 2019, de http://www.coordinadoradelamujer.org.bo/observatorio/archivos/marco/5copen80_562.pdf

Cruz, S. (2014). "Violencia y jóvenes: pandilla e identidad masculina en Ciudad Juárez”. Revista Mexicana de Sociología, 76(4). Pp. 613-637.

Dena, M. (2018). "Variables incidentes en la trata de personas. El cso de mujeres adolescentes en el Estado de Chihuahua”. Revista Latinoamericana de Derechos Humanos, 29(1). Pp. 139165.

Diaz, F., \& Reséndiz, A. (2017). "Factores de resiliencia y vulnerabilidad en jóvenes afectados por la violencia en Ciudad Juá- 
rez, Chihuahua. Argumentos, Estudios Críticos de la Sociedad, 30(84). Pp. 147-168.

Douzinas, C. (2006). "El fin(al) de los derechos humanos". Revista IUS, 7(1). Pp. 309-340.

Dworkin, A. (1997). Life and Death. Nueva York: Free Press.

Espinosa, D. (2015). Grupos en situación de vulnerabilidad. México: CNDH México.

Espinoza, R. (2019). "Violencia contra la mujer. ¿Un problema de normatividad penal o socio cultural?” Vox Juris, 37(12). Pp. 177-189.

Esteves, A. (2017). "La violencia contra las mujeres y la crisis de derechos humanos de la narcoguerra a las guerras necropolíticas". Estudios de Género de El Colegio de México, 3(6). Pp. 69-100.

Faci, A., \& Victory, A. (2017). "Los derechos culturales y los derechos de ls mujeres son derechos humanos y como tales deben ser gozados en igualdad". Revista Europea de Derechos Fundamentales, (29). Pp. 47-70.

Falk, R. (2002). "La globalización depredadora: una crítica”. Recensiones, Revista del Ministerio del Trabajo y Asuntos Sociales, (56). Pp. 236-239.

Fernández, A., Torres, A., \& Ochoa, G. (2019). “Discriminación de ingresos por género en un estado del norte de México: el caso Chihuahua”. Contexto, (8). Pp. 70-85.

Ferrajoli, L. (2006). "Sobre los derechos fundamentales. Cuestiones Constitucionales", Revista Mexicana de Derecho Constitucional, (15).

Gallegos, C., \& Rosales, G. (2012). "Epistemología Crítica”. Itinerario Educativo, (59). Pp. 15-29.

Galtung, J. (2016). "La violencia: cultural, estructural y directa". Cuadernos de estrategia, 183. Pp. 147-168.

Garzón, E. (1986). "Los deberes positivos generales y su fundamentación”. DOXA, (3). Pp. 17-33.

H. Congreso del Estado de Chihuahua 2018-2021 (2019). Unidad de Igualdad de Género. Obtenido de https://www.congresochihuahua.gob.mx/unidadGenero/unidad.php 
Herrera, P. (2000). "Rol de género y funcionamiento familiar". Revista Cubana de Medicina General Integral, 16(6). Pp. 568-573.

Hu, L., \& Bentler, P. (1999). "Cutoff criteria for fit indexes in covariance structure analysis: Conventional criteria versus new alternatives". Structural Equation Modeling: A Multidisciplinary Journal, 6(1). Pp. 1-55.

Inegi (Instituto Nacional de Estadística y Geografía) (2019). Reporte de defunciones por homicidio. Recuperado el 29 de Noviembre de 2019, de https://www.inegi.org.mx/sistemas/olap/ proyectos/bd/continuas/mortalidad/defuncioneshom.asp?s=est

--- (2016). Encuesta Nacional sobre la Dinámica de las Relaciones en los Hogares . Recuperado el 20 de abril de 2020, de https:// www.inegi.org.mx/contenidos/programas/endireh/2016/doc/ endireh2016_presentacion_ejecutiva.pdf

Instituto Nacional de las Mujeres (2007). Ley General de Accesos de las Mujeres a una Vida libre de Violencia (Primera edición ed.). México, D. F.: Inmujeres.

---. (2019). La paridad de género en todo un parteaguas para impulsar la transformación de México con igualdad inclusion y no discriminacion. Obtenido de https://www.gob.mx/inmujeres/prensa/la-paridad-de-genero-en-todo-un-parteaguas-paraimpulsar-la-transformacion-de-mexico-con-igualdad-inclusiony-no-discriminacion

Amnistía Internacional (2018). Historia de los derechos humanos. Recuperado el 29 de marzo de 2020, de Iusnaturalismo y positivismo: http://www.amnistiacatalunya.org/edu/es/historia/ inf-intro3.html

Lagarde, M. (2005). El Feminicidio, delito contra la humanidad. En Feminicidio, justicia y derecho. México: Comisión especial para Conocer y dar seguimiento a las investigaciones Relacionadas con los Feminicidios en la República Mexicana y a la Procuración de Justicia Vinculada.

---. (2007). "Por los derechos humanos de las mujeres: la Ley General de Acceso de las Mujeres a una Vida Libre de Violencia”. Revista mexicana de ciencias políticas y sociales, 49(200). Pp. 143-165. 
Lamas, M. (1996). "La perspectiva de género”. Revista de Educación y Cultura, 47. Pp. 216-229.

Lara, D. (2013). Grupos en situación de vulnerabilidad (Primera ed.). México, D. F.: Comisión Nacional de los Derechos Humanos.

Laurenzo, P. (2012). "Apuntes sobre el feminicidio". Revista de Derecho Penal y Criminología, 3(8). Pp. 119-143.

Loeza, L. (2017). "Violencia estructural, marcos de interpretación y derechos humanos en México”. Argumentos, 30(83). Pp. 249274.

López, M. (2009). "Los derechos humanos: ¿límites a la soberanía?" SCIO(4). Pp. 81-102.

Magallón, C. (2005). "Epistemología y violencia. Aproximación a una visión integral sobre la violencia hacia las mujeres". Feminismo/s (6). Pp. 33-47.

Maldonado, C. (2010). Hacia una fundamentación filosófica de los derechos humanos (Tercera ed.). Bogotá: Universidad del Rosario.

Martínez, M., \& Elizondo, F. (2018). La protección de los grupos en situación de vulnerabilidad en el Sistema Interamericano de Derechos Humanos (Primera ed.). Monterrey: Publicaciones UANL.

Masters, C. (2009). "Femina Sacra: The War on/of Terror, Women and the Femenine". Security Dialogue, 40(1). Pp. 29-49.

Meyen, M., Brewer, S., \& Cepeda, C. (2010). Abuso y miedo en Ciudad Juárez, un análisis de violaciones a los derechos humanos cometidas por militares en México. Recuperado el 12 de octubre de 2020, de https://www.wola.org/es/analisis/abuso-ymiedo-en-ciudad-juarez/

Monárrez, J. (2000). "La cultura del feminicidio en Ciudad Juárez, 1993-1999”. Frontera Norte, 12(23). Pp. 87-117.

---. (2004). Elementos de análisis del feminicidio sexual sistémico en Ciudad Juárez para su viaibilidad jurídica. Recuperado el 10 de junio de 2019, de http://mujeresdeguatemala.org/wpcontent/uploads/2014/06/Elementos-del-feminicidio-sexualsiste\%CC\%81mico.pdf 
---. (2004). Elementos de análisis del feminicidio sexual sistémico en Ciudad Juárez para su viaibilidad jurídica. Recuperado el 10 de junio de 2019, de http://mujeresdeguatemala.org/wpcontent/uploads/2014/06/Elementos-del-feminicidio-sexualsiste\%CC\%81mico.pdf

---. (2009). Trama de una injusticia: feminicidio sexual sistémico en Ciudad Juárez. México: El Colegio de la Frontera Norte.

---. (2010). Trama de una injustica: feminicidio sexual sistémico en Ciudad Juárez. México: El Colegio de la Frontera Norte y Miguel Angel Porrúa.

Naciones Unidas (10 de diciembre de 1948). La Declaración Universal de Derechos Humanos. Obtenido de La Declaración Universal de Derechos Humanos: http://www.un.org/es/universaldeclaration-human-rights/

---. (1993). Declaración sobre la eliminación de la violencia contra la mujer. Recuperado el 11 de junio de 2019, de https://www. ohchr.org/sp/professionalinterest/pages/violenceagainstwomen.aspx

--- (1994). Informe de la Conferencia Internacional sobre la Poblacióny el Desarrollo El Cairo. Nueva York: Naciones Unidas.

---. (1995). Cumbre Mundial Sobre Desarrollo Social, Copenhague. Recuperado el 18 de noviembre de 2019, de ://www.semujeres.cdmx.gob.mx/storage/app/media/DerechosMujeresyNinas/ Cumbre_DesarrolloSocialCopenhague.pdf

---. (2000). Beijing +5 Mujer 2000. Recuperado el 5 de diciembre de 2019, de https://www.un.org/es/events/pastevents/beijing_ plus_5/

---. (2018). Derecho Internacional de los Derechos Humanos. Recuperado el 20 de mayo de 2019, de http://www.hchr.org.mx/ index.php?option=com_content\&view=article\&id=452\&Item id $=250$.

Naciones Unidas, Derechos Humanos Oficina del Alto Comisionado (2006). Convención Internacional para la protección de todas las personas contra las desapariciones forazadas. Recuperado el 13 de junio de 2019, de https://www.ohchr.org/SP/ ProfessionalInterest/Pages/ConventionCED.aspx 
---. (1993). Declaración sobre la eliminación de la violencia contra la mujer. Recuperado el 05 de enero de 2019, de https:// www.ohchr.org/sp/professionalinterest/pages/violenceagainstwomen.aspx

--- (2019). Derecho Internacional de los Derechos Humanos. Recuperado el 23 de Septiembre de 2019, de https://www.hchr. org.mx/index.php?option=com_content\&view=article\&id=452 \&Itemid $=250$

Naciones Unidas, CEPAL (2016). Autonomía de las mujeres e igualdad en la agenda de desarrollo sostenible. Recuperado el 5 de abril de 2020, de https://repositorio.cepal.org/bitstream/handle/11362/40633/S1601248_es.pdf?sequence=4\&isAllowed=y

Naciones Unidas, Oficina del Alto Comisionado (1993). Conferencia Mundial de Derechos Humanos Viena. Recuperado el 7 de diciembre de 2019, de https://www.ohchr.org/SP/NewsEvents/ OHCHR20/Pages/WCHR.aspx

Nelson, W. (1981). "Human rights and human obligations". Nomo American Society for Political and Legal Philosophy, 23. Pp. 281-296.

Nikken, P. (1994). El Concepto de Derechos Humanos. San José: IIDH.

Observatorio Ciudadano de los Derechos de las Mujeres (2019). ¿Qué es la CEDAW? Recuperado el 14 de diciembre de 2019, de https://catedraunescodh.unam.mx/catedra/mujeres3/CEDAW2/index2b9e.html?option=com_content\&view=article\&id $=1 \&$ Itemid $=2$

Oficina del Alto Comisionado de Derechos Humanos de las Naciones Unidas. (16 de diciembre de 1966). Pacto Internacional de Derechos Civiles y Políticos. Recuperado el 28 de febrero de 2019, de https://www.ohchr.org/sp/professionalinterest/pages/ ccpr.aspx

---. (18 de diciembre de 1979). Convención sobre la eliminación de todas las formas de discriminación contra la mujer. Recuperado el 27 de febrero de 2019, de https://www.ohchr.org/sp/ professionalinterest/pages/cedaw.aspx 
---. (18 de diciembre de 1979). Convención sobre la eliminación de todas las formas de discriminación contra la mujer. Recuperado el 27 de febrero de 2019, de https://www.ohchr.org/sp/ professionalinterest/pages/cedaw.aspx

ONU Mujeres (2011). UNIFEM Plan estratégico. Recuperado el 7 de diciembre de 2019, de https://www.unwomen.org/ es/digital-library/publications/2011/3/unifem-strategic-plan2008-2011-evaluability-assessment\#view

---. (2016). Conferencias mundiales sobre la mujer. Recuperado el 10 de diciembre de 2019, de https://www.unwomen.org/es/ how-we-work/intergovernmental-support/world-conferenceson-women

---. (2019). Un poco de historia. Recuperado el 12 de junio de 2019, de http://www.unwomen.org/es/csw/brief-history

--- (2020). Violencia contra las mujeres y niñas. Recuperado el 22 de abril de 2020, de https://trainingcentre.unwomen.org/mod/ scorm/player.php?a=169\&currentorg=Yo_s?_de_G?nero._Una_ introducci?n_a_la_Igualdad_de_G?nero_para_la_ONU_personal_ORG\&scoid=372\&sesskey=PxC5ULSf2G\&display=popup\& mode $=$ normal

ONU Mujeres México. (1979). Convención sobre la Eliminaciónde todas las formas de Discriminación contra la Mujer. Recuperado el 13 de junio de 2019, de http://mexico.unwomen.org/es/ digiteca/publicaciones/2011/12/cedaw

ONU Mujeres, Secretaría de Gobernación, Instituto Nacional de las Mujeres (2017). La violencia feminicida en México, aproximaciones y tendencias 1985-2016. Recuperado el 10 de abril de 2020, de https://www2.unwomen.org/-/media/field\%20office\%20mexico/documentos/publicaciones/2017/10/violenciafeminicidamx\%2007dic\%20web.pdf?la=es\&vs=5302

Organización de Estados Americanos (22 de noviembre de 1969). Convención Americana sobre Derechos Humanos. Recuperado el 28 de febrero de 2019, de https://www.oas.org/dil/esp/ tratados_b-32_convencion_americana_sobre_derechos_humanos.htm 
---. (9 de junio de 1994). Convención Interamericana para prevenir, sancionar y erradicar la violencia contra la mujer "Convención de Belem Do Para. Recuperado el 28 de febrero de 2019, de http://www.oas.org/juridico/spanish/tratados/a-61.html

---. (9 de junio de 1994). Convención Interamericana para prevenir, sancionar y erradicar la violencia contra la mujer "Convención de Belem Do Para. Recuperado el 28 de febrero de 2019, de http://www.oas.org/juridico/spanish/tratados/a-61.html

---. (mayo de 2017). Ley Modelo Interamericana sobre Violencia Política contra las Mujeres. Recuperado el 28 de febrero de 2019, de http://blog.pucp.edu.pe/blog/fernandotuesta/wp-content/uploads/sites/945/2017/05/Ley-modelo-Violencia-contraMujer.pdf

Organización de las Naciones Unidas (2011). Convención sobre la Eliminación de todas formas de Discriminación contra la Mujer. Recuperado el 11 de junio de 2019, de http://mexico. unwomen.org/es/digiteca/publicaciones/2011/12/cedaw

Organización Mundial de la Salud (2017). Violencia contra la mujer. Recuperado el 12 de junio de 2019, de https://www.who. int/es/news-room/fact-sheets/detail/violence-against-women

--- (2020, p. 1). Temas de la Salud. Recuperado el 18 de abril de 2020, de https://www.who.int/topics/violence/es/

Organización de Estados Americanos, Departamento de Derecho Internacional (1994). Convención Interamericana para Prevenir, Sancionar y Erradicar la Violencia Contra la Mujer "Convención de Belem do Pará". Recuperado el 13 de junio de 2019, de https://www.oas.org/juridico/spanish/tratados/a-61.html

Pinto, M. (2017). “Discriminación y violencia. Un comentario sobre los derechos de las mujeres en el marco internacional de los derechos humanos". En U. d. Aires (Ed.), Pensar en Derech. Buenos Aires: Eudeba.

Pizarro, J. (2017). "Feminismos, perspectiva de género y psicoanálisis”. Revista GénEros, 2(20). Pp. 35-63.

Polo, L. (junio de 20 de 2000). Fundamentos filosóficos de los derechos humanos. Obtenido de http://www.url.edu.gt/PortalURL/ Archivos/83/Archivos/Departamento\%20de\%20Investigacio- 
nes\%20y\%20publicaciones/Articulos\%20Doctrinarios/Derecho/ Fundamentos\%20filosoficos\%20de\%20los\%20Derechos\%20Humanos.pdf

Radford, J., \& Rusell, D. (1992). Femicide: The Politics of Woman Killing. Nueva York: Twayne Publishers.

Ravelo, P. (2005). "La costumbre de matar: proliferación de la violencia en Ciudad Juárez, Chihuahua, México”. Nueva antropología, 20(65). Pp. 149-166.

Red TDT (2017). Violencia contra las mujeres, un problema histórico. Recuperado el 13 de junio de 2019, de https://redtdt.org. $\mathrm{mx} /$ violencias_mujeres/index.php/el-informe/violencia-contralas-mujeres-un-problema-historico/

Rodríguez, J. (2000). Vulnerabilidad Demográfica en Bolivia, Ecuador, Nicaragua y Uruguay (Primera ed.). Santiago de Chile: Comisión Econámica para America Latina y el Caribe.

Rodríguez, T. (2005). Relatoría de la segunda mesa "Seguimiento a los informes internacionales sobre Ciudad Juárez, del Seminario Internacional: Feminicidio, Justicia y Derecho. En Feminicidio, justicia y derecho México, D.F.: Comisión Especial para Conocer y dar Seguimiento a las Investigaciones Relacionadas con los Feminicidios en la República Mexicana y a la Procuración de Justicia Vinculada.

Román, J. (8 de octubre de 2015). Propone el visitante cronograma para retirar al ejército de tareas policiacas. Recuperado el 12 de abril de 2010, de https://www.jornada.com.mx/2015/10/08/ politica/003n2pol

---. (23 de febrero de 2017). Vive México la peor crisis de derechos humanos, acusa Amnistía Internacional, La jornada, obtenido de: https://www.jornada.com.mx/2017/02/23/politica/012n1pol

Rusell, D. (2005). “Definición de feminicidio y conceptos relacionados". En Feminicidio, justicia y derecho. México, D.F.: Comisión Especial para Conocer y dar Seguimiento a las Investigaciones Relacionadas con los Feminicidios en la República Mexicana.

Secretaría de Relaciones Exteriores (5 de julio de 2012). Igualdad entre hombres y mujeres. Recuperado el 28 de febrero de 2019, 
de Igualdad entre hombres y mujeres: https://www.gob.mx/ sre/acciones-y-programas/igualdad-entre-mujeres-y-hombres Secretariado Ejecutivo del Sistema Nacional de Seguridad Pública (2019). Incidencia delictiva del fuero común. Recuperado el 5 de enero de 2020, de https://drive.google.com/ file/d/17i5n6Kfs9CvXAYgBZ-UCDNBK1pA_btP6/view

Segato, R. (2003). "La argamasa jerárquica: violencia moral, reproducción del mundo y la eficacia simbolica del Derecho". En M. I. Silberg (Ed.), Las estructuras elementales de la violencia. Buenos Aires: Universidad Nacional de Quilmes Editorial.

---. (2003). "Las estructuras elementales de la violencia: contrato y estatus en la etiología de la violencia”. En Primera (Ed.), Las estructuras elementales de la violencia: Ensayos sobre género entre la antropología, el psicoanálisis y los derechos humanos. Buenos Aires: Bernal.

---. (2004). Territorio, soberanía y crímenes de segundo Estado: La escritura en el cuerpo de las mujeres asesinadas en Ciudad Juárez. En N. H. Ciudad Juárez: de este lado del puente. México: Ekipedia.

Semelin, J. (1983). Para salir de la violencia. París: The Works Editions.

Straudt, K., \& B., V. (2006). "Mujeres, políticas públicas y política: los caminos globales de Ciudad Juárez, Chihuahua-El Paso, Texas". Región y sociedad, 18(37). Pp. 127-172.

Suprema Corte de Justicia de la Nación; Universidad Nacional Autónoma de México; Adenauer, Fundación Konrad (2013). Derechos Humanos en la Constitución: Comentarios de Jurisprudencia Constitucional e Interamericana (Primera ed.). México, D.F.: Coordinación de Compilación y Sistematización de Tesis de la Suprema Corte de Justicia de la Nación de México. Torres, E. (2018). "Mujeres protagonistas de nuestra historia”. Ambigua, Revista de Investigaciones sobre Género y Estudios Culturales, 114-117.

Tribunal Electoral del Poder Judicial de la Federación (2019). Protocolo para Atender la Violencia Política contra las Mujeres (Primera ed.). Ciudad de México: Editorial TE. 
United Nations Women (2019). Wolrld Conferences on women. Recuperado el 5 de diciembre de 2019, de https://www.unwomen.org/es/how-we-work/intergovernmental-support/worldconferences-on-women

Villegas, P. (6 de enero de 2020). A New Toll in Mexico's Drug War: More Than 61,000 Vanished. The New York Times, https:// www.nytimes.com/2020/01/06/world/americas/mexico-drugwar-death-toll.html.

Williams, T. (mayo de 18 de 2002). Fundamentos de los Derechos del Hombre y el Principio Rector del Bien Común. Obtenido de http://www.catolicos.com/socialdoc12.htm.

Zúñiga, M., Bejarano, M., Aranda, P., \& Arellano, C. (2012). Región noroeste: Baja California, Baja California Sur, Sinaloa y Sonora. En Primera (Ed.), Estudio nacional sobre las fuentes, orígenes y factores que producen y reproducen la violencia contra las mujeres. México, D. F.: CONAVIM-CRIM-UNAM. 